\title{
Feeding Babies and Bugs
}

\author{
Kjersti Aagaard
}

Keywords: microbiome, delivery type, metagenomes

A LTHOUgh GENOMIC VARIATION and epigenomics contribute to metabolic programming, the in utero environment, particularly the perinatal and early metagenomes, can shape our metabolic programming in diverse, resilient, and rather creative ways. Accumulating evidence suggests that the in utero environment is not sterile; several groups have reported evidence of bacteria in the placenta, amniotic fluid, cord blood, and meconium. ${ }^{1-4}$ One study also demonstrated that neonates harbor microbial communities that have been shown to be relatively homogenous at delivery, with the exception of the meconium, which contains microbes also detected in the placenta and amniotic fluid (Fig. 1). ${ }^{5}$ By 6 weeks of age, the infant microbiota structure and function expand and differentiate by body site, similar to the maternal microbiota. The neonatal microbiota structure also varies by mode of delivery in some body sites (oral cavity, nares, and skin), but there are no differences in microbiota structure or function at 6 weeks, suggesting that there is no persistent impact of cesarean delivery on the infant microbiota. 5
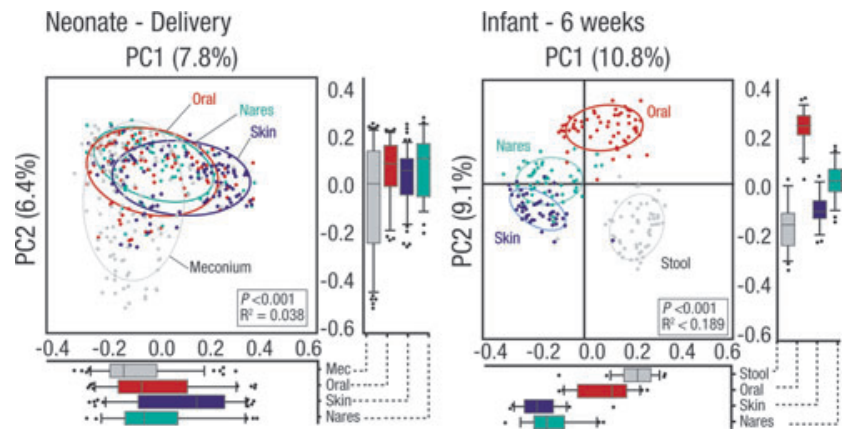

FIG. 1. Differences in the neonatal and infant (6 weeks of age) microbial communities. Adapted from Chu et al., 2017.
Although the mode of delivery does not seem to significantly alter the infant microbiome, other factors, such as maternal diet during pregnancy, may have a lasting impact. Studies in nonhuman primates have demonstrated that a high-fat maternal diet during gestation and through lactation can persistently alter the gut microbiome in offspring at 1 year of age. ${ }^{6,7}$ Specifically, in this study, offspring exposed to a maternal high-fat diet had a significantly lower abundance of Campylobacter than offspring born to mothers on a low-fat control diet. The resultant dysbiosis was not fully corrected when offspring were weaned onto a control diet (Fig. 2). ${ }^{6}$ Current studies are assessing whether a maternal high-fat diet during pregnancy and lactation has any impact on offspring behavior. Other studies are also investigating whether the maternal diet can alter the structure of the breast milk microbiome and/or other bioactive components (e.g., human milk oligosaccharides).

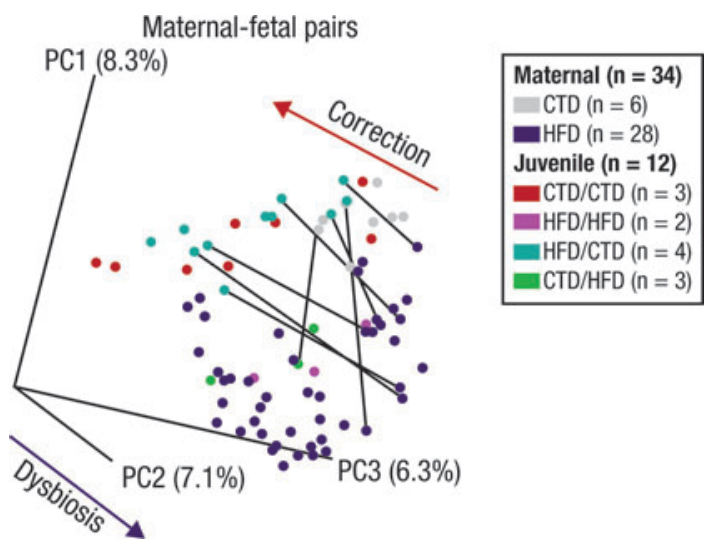

FIG. 2. Gestational influence of the maternal diet is not fully correctable with a normal postnatal diet. CTD, control diet; HFD, high-fat diet. Adapted from Ma et al., 2014. ${ }^{6}$

Division of Maternal-Fetal Medicine (MFM), Department of Obstetrics and Gynecology, Baylor College of Medicine and Texas Children's Hospital, Houston, Texas.

(C) Kjersti Aagaard, 2019; Published by Mary Ann Liebert, Inc. This Open Access article is distributed under the terms of the Creative Commons License (http://creativecommons.org/licenses/by/4.0), which permits unrestricted use, distribution, and reproduction in any medium, provided the original work is properly cited. 
Together, these findings are helping to challenge the long-standing paradigm of a sterile uterine environment. Low biomass communities (e.g., those in breast milk or the uterine environment) may influence the development of metabolic and immune diseases later in life. The maternal diet has been shown to be a potent modulator of the offspring gut microbiome and may have long-term developmental consequences. Determining what factors shape the microbiome during infancy and throughout the lifespan will help to further the understanding of how the microbiome may impact development and disease in later life.

\section{Disclosure Statement}

No competing financial interests exist.

\section{References}

1. Aagaard K, Ma J, Antony KM, et al. The placenta harbors a unique microbiome. Sci Transl Med 2014;6:237ra65.

2. Mueller NT, Bakacs E, Combellick J, et al. The infant microbiome development: Mom matters. Trends Mol Med 2015;21:109-117.

3. Gomez-Arango LF, Barrett HL, David McIntyre H, et al. Contributions of the maternal oral and gut microbiome to placental microbial colonization in overweight and obese pregnant women. Sci Rep 2017;7:2860.

4. Chen C, Song X, Wei W, et al. The microbiota continuum along the female reproductive tract and its relation to uterine-related diseases. Nat Commun 2017;8:875.

5. Chu DM, Ma J, Prince AL, et al. Maturation of the infant microbiome community structure and function across multiple body sites and in relation to mode of delivery. Nat Med 2017;23:314-326.

6. Ma J, Prince AL, Bader D, et al. High-fat maternal diet during pregnancy persistently alters the offspring microbiome in a primate model. Nat Commun 2014;5:3889.

7. Pace RM, Prince AL, Ma J, et al. Modulations in the offspring gut microbiome are refractory to postnatal synbiotic supplementation among juvenile primates. BMC Microbiol 2018;18:28.

Address correspondence to: Kjersti Aagaard, MD, PhD, FACOG Division of Maternal-Fetal Medicine (MFM) Department of Obstetrics and Gynecology Baylor College of Medicine and Texas Children's Hospital Houston, TX 77030

E-mail: aagaardt@bcm.edu 